Homology, Homotopy and Applications, vol.4(2), 2002, pp.409-426

\title{
SOME CONJECTURES ABOUT THE HILBERT SERIES OF GENERIC IDEALS IN THE EXTERIOR ALGEBRA
}

\author{
GUILLERMO MORENO-SOCÍAS AND JAN SNELLMAN
}

(communicated by Larry Lambe)

\begin{abstract}
We calculate the Hilbert series of a quotient of the exterior algebra by a generic form of even degree, and give conjectures about the Hilbert series of other generic quotients.
\end{abstract}

To Jan-Erik Roos on his sixty-fifth birthday

\section{Introduction}

In the symmetric algebra $K\left[x_{1}, \ldots, x_{n}\right]$, the set of Hilbert series coming from homogeneous quotients are classified by Macaulays theorem $[\mathbf{1 6}, \mathbf{6}, \mathbf{3}]$. There is an infinite number of possible series, but if we fix positive integers $d_{1}, \ldots, d_{r}$, and restrict our study to quotients by homogeneous ideals $I$ of "type" or "numerical character" $\left(d_{1}, \ldots, d_{r}\right)$, ie generated by forms of those prescribed degrees, then there are only finitely many Hilbert series. Furthermore, in the affine space parametrising these homogeneous ideals, there is a Zariski-open subset of ideals with the same Hilbert series, and the Hilbert series obtained on this open set is minimal $[\mathbf{9}, \mathbf{7}]$.

Unfortunately, even though we know the set of all Hilbert series, we do not know what Hilbert series arise from ideals of numerical character $\left(d_{1}, \ldots, d_{r}\right)$. In fact, we do not even know the "generic" series, but it is conjectured $[\mathbf{1 7}, \mathbf{7}]$ that it is $\left\langle(1-t)^{-n} \prod_{i=1}^{r}\left(1-t^{d_{i}}\right)\right\rangle$; the brackets mean "truncate before the first non-positive coefficient".

In the exterior algebra $\bigwedge V_{n}$, we also know the set of all Hilbert series of homogeneous quotients, by the so-called Kruskal-Katona theorem $[\mathbf{1 5}, \mathbf{1 4}, \mathbf{5}, \mathbf{2}]$. Here, this set is finite, so one would think that it should be easy to find the subset of Hilbert series coming from quotients by ideals having a prescribed numerical character. In particular, it should be easy to find the generic value. However, very little is known.

In this article, we give one new result (the series for a quotient by one form of even degree) and several conjectures, supported by extensive computer calculations.

It is worthwhile to point out that the problem of determining the Hilbert series of quotients by generic quadratic forms is especially interesting, since it determines

Snellman was supported grants from Svenska institutet and by grant $n$. $231801 \mathrm{~F}$ from Centre International des Etudiants et Stagiaires while visiting cole Polytechnique, and by grants from Svenska Institutet and Kungliga Vetenskapsakademin while visiting University of Wales, Bangor Received December 15, 2000, revised March 16, 2001; published on July 12, 2002.

2000 Mathematics Subject Classification: 15A75, 13D40.

Key words and phrases: exterior algebra, generic ideals, Hilbert series.

(c) 2002, Guillermo Moreno-Socías and Jan Snellman. Permission to copy for private use granted. 
the Koszulness of the quadratic algebras in question. We refer to the recent article by Fröberg and Löfwall [10].

\section{Notation}

Let $K$ be a field of characteristic 0 . Then $\mathbb{Q}$ is the prime subfield of $K$. For any positive integer $n$, let $V=V_{n}$ be an $n$-dimensional vector space over $K$, with a distinguished basis $X_{n}=\left\{x_{1}, \ldots, x_{n}\right\}$. Let $K\left[x_{1}, \ldots, x_{n}\right]$ denote the symmetric algebra on $V_{n}$, and let $\bigwedge V_{n}$ denote the exterior algebra on $V_{n}$. We define $\mathfrak{S}\left(V_{n}\right)$, the square-free algebra on $V_{n}$, to be the commutative $K$-algebra generated by $X_{n}$, with the relations $x_{i}^{2}=0$; in other words, $\mathfrak{S}\left(V_{n}\right)=\frac{K\left[x_{1}, \ldots, x_{n}\right]}{\left(x_{1}^{2}, \ldots, x_{n}^{2}\right)}$. There is an isomorphism of graded vector spaces between $\bigwedge V_{n}$ and $\mathfrak{S}\left(V_{n}\right)$, but they are not isomorphic as $K$ algebras, since the exterior algebra is skew-commutative and $\mathfrak{S}\left(V_{n}\right)$ is commutative.

We shall need the following operations for formal power series.

Definition 2.1. Let $f(t)=\sum_{i=0}^{\infty} a_{i} t^{i} \in \mathbb{Z}[[t]], g(t)=\sum_{i=0}^{\infty} b_{i} t^{i} \in \mathbb{Z}[[t]]$. We say that $f \geqslant g$ if $a_{i} \geqslant b_{i}$ for all $i$. We define

$$
\begin{aligned}
\max (f(t), g(t)) & =\sum_{i=0}^{\infty} \max \left(a_{i}, b_{i}\right) \\
\langle f(t)\rangle & =\sum_{i=0}^{\ell} a_{i} t^{i}, \quad \ell=\max \left(\left\{i \mid a_{j}>0 \text { for } j \leqslant i\right\}\right) \\
\rangle f(t)\langle & =\sum_{i=\ell}^{\infty} a_{i} t^{i}, \quad \ell=\min \left(\left\{i \mid a_{j}>0 \text { for } j \geqslant i\right\}\right)
\end{aligned}
$$

We use the conventions $\max (\emptyset)=-1=\min (\mathbb{N}), \min (\emptyset)=+\infty=\max (\mathbb{N})$.

Let $\left[X_{n}\right]$ denote the free abelian monoid on $X_{n}$, and denote by $Y_{n}$ the subset of square-free monomials. Then $Y_{n}$ is a $K$-basis for both $\bigwedge V_{n}$ and $\mathfrak{S}\left(V_{n}\right)$. We define the degree of a monomial in $\left[X_{n}\right]$ (and in $Y_{n}$ ) in the usual way, and denote by $\left[X_{n}\right]^{d}$ and $Y_{n}^{d}$ the subset of monomials (square-free monomials) of degree $d$.

A form $\bigwedge K\left[x_{1}, \ldots, x_{n}\right] \ni f=\sum_{m \in\left[X_{n}\right]^{d}} c_{m} m$ is said to be generic if the coefficients $c_{m} \in K$ fulfil the following conditions:

1. $c_{m} \notin \mathbb{Q}$,

2. $m \neq m^{\prime} \Longrightarrow c_{m} \neq c_{m^{\prime}}$,

3. The set of all $c_{m}$ 's is algebraically independent over $\mathbb{Q}$.

A homogeneous ideal $I \subset K\left[x_{1}, \ldots, x_{n}\right]$ is called generic if it can be minimally generated by a finite set of generic forms, so that all of the occuring coefficients of the forms are different, and so that the set of all occuring coefficients is algebraically independent over $\mathbb{Q}$. If the forms have degrees $d_{1}, \ldots, d_{r}$, then we say that $I$ has "numerical character" $\left(d_{1}, \ldots, d_{r}\right)$. It is an important fact that any two generic ideals of the same numerical character have the same initial ideal and the same Hilbert series. 
Now consider the affine space $V=\mathbf{A}^{\left(\begin{array}{c}n+d_{1}-1 \\ d_{1}\end{array}\right)} \times \cdots \times \mathbf{A}^{\left(\begin{array}{c}n+d_{r}-1 \\ d_{r}\end{array}\right)}$ parametrising the set of homogeneous ideals of numerical character $\left(d_{1}, \ldots, d_{r}\right)$. Since there are countably many conditions to be fulfilled for an ideal to be generic, the subset of the parameter space corresponding to generic ideals is not open, but a countable intersection of open sets, hence dense. However, in $V$ there is a Zariski-open subset corresponding to ideals with the same Hilbert function, and the generic ideals are contained in this subset $[\mathbf{9}]$.

We make similar definitions for the square-free algebra, and for the exterior algebra. Here, a generic form is a generic linear combination of square-free monomials of a certain degree. It is still true that the generic Hilbert series is attained on an open component of the parameter space, and that the generic ideals are contained in this component.

\section{Hilbert series for generic principal ideals in the symmetric and square-free algebra}

\subsection{Principal ideals in the symmetric algebra}

If $f \in K\left[x_{1}, \ldots, x_{n}\right]$ is a non-zero form of degree $d$, not necessarily homogeneous, then clearly the Hilbert series of the quotient $\frac{K\left[x_{1}, \ldots, x_{n}\right]}{(f)}$ is $(1-t)^{-n}\left(1-t^{d}\right)$.

\subsection{Principal ideals in the square-free algebra}

If $f \in \mathfrak{S}\left(V_{n}\right)$ is a generic form of degree $d$, then there is a similar simple formula for $\frac{\mathfrak{S}\left(V_{n}\right)}{(f)}(t)$ (the Hilbert series of the quotient). To state the formula, we need some additional notation.

Definition 3.1. We denote the zero series by 0 , and define

$$
\begin{aligned}
\Delta_{n, d}(t) & =\rangle\left(t^{d}-1\right)(1+t)^{n}\langle \\
& =\sum_{v=\lceil(n-d) / 2\rceil}^{n}\left(\left(\begin{array}{l}
n \\
v
\end{array}\right)-\left(\begin{array}{c}
n \\
v+d
\end{array}\right)\right) t^{v} \\
\delta_{n, d}(t) & =\left\langle(1+t)^{n}\left(1-t^{d}\right)\right\rangle \\
& =\sum_{v=0}^{\lfloor(n-d) / 2\rfloor}\left(\left(\begin{array}{c}
n \\
v+d
\end{array}\right)-\left(\begin{array}{l}
n \\
v
\end{array}\right)\right) t^{v}
\end{aligned}
$$

The following result is due to Frberg [8].

Theorem 3.2. Let $f \subset \mathfrak{S}\left(V_{n}\right)$ be a generic form of degree $d$. Then

$$
\frac{\mathfrak{S}\left(V_{n}\right)}{(f)}(t)=\delta_{n, d}(t)
$$

Proof. By considering the graded exact sequence

$$
0 \longrightarrow \operatorname{ann}(f)(-d) \longrightarrow \mathfrak{S}\left(V_{n}\right)(-d) \stackrel{\cdot f}{\longrightarrow} \mathfrak{S}\left(V_{n}\right) \longrightarrow \frac{\mathfrak{S}\left(V_{n}\right)}{(f)} \longrightarrow 0
$$


in each degree $r$, we see that (1) holds if and only if multiplication by $f$, regarded as a linear map $\phi_{r}$ from $\mathfrak{S}\left(V_{n}\right)_{r}$ to $\mathfrak{S}\left(V_{n}\right)_{r+d}$, is injective when $\left(\begin{array}{c}n \\ r\end{array}\right) \leqslant\left(\begin{array}{c}n \\ r+d\end{array}\right)$, and surjective when $\left(\begin{array}{c}n \\ r\end{array}\right) \geqslant\left(\begin{array}{c}n \\ r+d\end{array}\right)$.

Write $f=\sum_{m \in Y_{n}^{d}} c_{m} m$. For $0 \leqslant r \leqslant n-d, Y_{n}^{r}$ is a basis of $\mathfrak{S}\left(V_{n}\right)_{r}$, and $Y_{n}^{r+d}$ is a basis of $\mathfrak{S}\left(V_{n}\right)_{r+d}$. Thus, we must show that for each $r$, the matrix of $\phi_{r}$ in this basis has maximal rank. This matrix has rows indexed by $Y_{n}^{r+d}$ and columns indexed by $Y_{n}^{r}$. The entry at row $R$, column $C$ is

$$
\begin{cases}0 & C \backslash R \\ c_{m} & R=m C\end{cases}
$$

If we specialise this matrix, the rank can only decrease, so if we can prove that some specialised matrix has full rank, then we are done. Putting all $c_{m}=1$, we obtain the incidence matrix of $r$-subsets of $[n]$ into $r+d$-subsets of $[n]$, that is, the rows are indexed by $r$-subsets and the columns by $r+d$-subsets, with a 1 at the $a, b$ 'th position iff $a \subset b$, and 0 otherwise. It has been shown by combinatorialists that this matrix has full rank $[\mathbf{1 8}, \mathbf{1 3}, \mathbf{1 1}]$.

\section{Principal ideals in the exterior algebra - the difference between even and odd degree}

Let $f \in \bigwedge V_{n}$ be a generic form of degree $d$. Denote the Hilbert series of $\frac{\bigwedge V_{n}}{f}$ by $q_{n, d}(t)$, that of the annihilator of $f$ by $a_{n, d}(t)$, and that of the principal ideal $(f)$ by $p_{n, d}(t)$. From the the graded exact sequence

$$
0 \longrightarrow \operatorname{ann}(f)(-d) \longrightarrow \bigwedge V_{n}(-d) \stackrel{\cdot f}{\longrightarrow} \bigwedge V_{n} \longrightarrow \frac{\bigwedge V_{n}}{(f)} \longrightarrow 0
$$

we get that

$$
\begin{aligned}
q_{n, d}(t) & =t^{d} a_{n, d}(t)-t^{d}(1+t)^{n}+(1+t)^{n} \\
& =t^{d} a_{n, d}(t)+(1+t)^{n}\left(1-t^{d}\right) \\
a_{n, d}(t) & =t^{-d}\left(q_{n, d}(t)-(1+t)^{n}\left(1-t^{d}\right)\right)
\end{aligned}
$$

If $d$ is even, we shall prove that the vector space map

$$
\bigwedge^{v} V_{n} \stackrel{\cdot f}{\rightarrow} \bigwedge^{v+d} V_{n}
$$

is injective "when it can be", ie when $\left(\begin{array}{l}n \\ v\end{array}\right) \leqslant\left(\begin{array}{c}n \\ v+d\end{array}\right)$, and surjective "when it can be", 
ie when $\left(\begin{array}{l}n \\ v\end{array}\right) \geqslant\left(\begin{array}{c}n \\ v+d\end{array}\right)$. This leads immediately to the formulæ

$$
\begin{aligned}
q_{n, d}(t) & =\left\langle(1+t)^{n}\left(1-t^{d}\right)\right\rangle=\delta_{n, d}(t) \\
a_{n, d}(t) & =t^{-d}\left(q_{n, d}(t)-\left(1-t^{d}\right)(1+t)^{n}\right) \\
& =t^{-d}\left(\delta_{n, d}(t)-\left(1-t^{d}\right)(1+t)^{n}\right) \\
& =t^{-d} \sum_{r=0}^{n}\left[\max \left(0,\left(\begin{array}{c}
n \\
r+d
\end{array}\right)-\left(\begin{array}{c}
n \\
r
\end{array}\right)\right)-\left(\left(\begin{array}{c}
n \\
r+d
\end{array}\right)-\left(\begin{array}{c}
n \\
r
\end{array}\right)\right)\right] t^{r} \\
& =t^{-d} \sum_{r=0}^{n} \max \left(0,-\left(\begin{array}{c}
n \\
r+d
\end{array}\right)+\left(\begin{array}{c}
n \\
r
\end{array}\right)\right) t^{r} \\
& =t^{-d} \Delta_{n, d}(t)
\end{aligned}
$$

In particular, as $n \rightarrow \infty,(1+t)^{-n} q_{n, d}(t) \rightarrow\left(1-t^{d}\right)$, and $a_{n, d}(t) \rightarrow 0$, with respect to the $t$-adic norm on $\mathbb{Z}[[t]]$.

If $d$ is odd, then we have that $f^{2}=0$, hence $f g=0$ whenever $g \in(f)$, hence $\operatorname{ann}(f) \supseteq(f)$, hence $a_{n, d}(t) \geqslant p_{n, d}(t)$. In other words, there is a graded complex

$$
(\bigwedge V)(-d) \stackrel{\cdot f}{\rightarrow} \bigwedge V \stackrel{\cdot f}{\longrightarrow}(\bigwedge V)(d)
$$

the graded homology of which determines $a_{n, d}(t)-p_{n, d}(t)$. In the (not very interesting) case $d=1$, then we know from [1] that this homology vanishes. For odd $d>1$, we guess that for a fixed degree $r$, and $n$ very large, this homology vanishes. Hence, in degree $r$, the "obstruction to injectivity" in (5) is as small as possible. An equivalent formulation: consider the start of a minimal free graded $\wedge V_{n}$-resolution of $\frac{\wedge V_{n}}{(f)}$

$$
\frac{\bigwedge V_{n}}{(f)} \leftarrow \bigwedge V_{n} \stackrel{\cdot f}{\longleftarrow} \bigwedge V_{n} \leftarrow \bigoplus_{j=1}^{r}\left(\bigwedge V_{n}\right)\left(-\beta_{2, i}\right)
$$

where $\beta_{2, i}$ are the graded Betti numbers. Then we guess that as $n$ increases, and for a fixed $i \neq 2 d, \beta_{2, i}=0$. On the other hand, for sufficiently large $n$, we guess that $\beta_{2,2 d}=1$. Since $\beta_{2, i}$ is the dimension of the degree $i-d$ part of a certain Tor group, this conjecture can also be stated in terms of Cartan homology (see [2]).

We show the order (ie the smallest $\ell$ for which $t^{\ell}$ occurs with non-zero coefficient) of $a_{n, d}(t)-p_{n, d}(t)$ for small $n, d$ in Table 1 . It would seem that the order of the difference grows linearly in $n$, so that $a_{n, d}(t)-p_{n, d}(t) \rightarrow 0$ rather rapidly.

Let us turn to the consequences of this conjecture. We get that $a_{n, d}(t) \sim p_{n, d}(t)$ with respect to the $(t)$-adic filtration. It then follows from (4) that

$$
q_{n, d}(t) \sim t^{d} p_{n, d}(t)+(1+t)^{n}\left(1-t^{d}\right)
$$

Substituting $p_{n, d}(t)=(1+t)^{n}-q_{n, d}(t)$ and solving for $q_{n, d}(t)$ we get that

$$
q_{n, d}(t) \sim \frac{(1+t)^{n} t^{d}+(1+t)^{n}\left(1-t^{d}\right)}{\left(1+t^{d}\right)}=\frac{(1+t)^{n}}{\left(1+t^{d}\right)},
$$




\begin{tabular}{|c|cccccccc|}
\hline $\mathrm{d}$ & 3 & 5 & 7 & 9 & 11 & 13 & 15 & 17 \\
\hline $\mathrm{n}$ & & & & & & & & \\
3 & 1 & & & & & & & \\
4 & 1 & & & & & & & \\
5 & 1 & 1 & & & & & & \\
6 & 2 & 1 & & & & & & \\
7 & 3 & 1 & 1 & & & & & \\
8 & 3 & 2 & 1 & & & & & \\
9 & 3 & 3 & 1 & 1 & & & & \\
10 & 4 & 3 & 2 & 1 & & & & \\
11 & 5 & 3 & 3 & 1 & 1 & & & \\
12 & 5 & 4 & 3 & 2 & 1 & & & \\
13 & 5 & 5 & 4 & 3 & 1 & 1 & & \\
14 & 6 & 5 & 4 & 3 & 2 & 1 & & \\
15 & 7 & 6 & 5 & 3 & 3 & 1 & 1 & \\
16 & 7 & 6 & 5 & 4 & 3 & 2 & 1 & \\
17 & - & - & - & 5 & 4 & 3 & 1 & 1 \\
18 & - & - & - & - & 4 & 3 & 2 & 1 \\
19 & - & - & - & - & - & 3 & 3 & 1 \\
20 & - & - & - & - & - & - & 3 & 2 \\
\hline
\end{tabular}

Table 1: Order of $a_{n, d}(t)-p_{n, d}(t)$ for small $n, d$

hence

$$
\frac{\frac{\bigwedge V_{n}}{(f)}(t)}{\bigwedge\left(V_{n}\right)(t)}=\frac{q_{n, d}(t)}{(1+t)^{n}} \rightarrow \frac{1}{1+t^{d}} \quad \text { as } n \rightarrow \infty
$$

\section{Principal ideals on generic forms of even degree in the exterior algebra}

If $d=2$ then we can change coordinates on $V$ and replace $f$ with the form $x_{1} x_{2}+x_{3} x_{4}+\cdots$, as is demonstrated in [4]. The Hilbert series of the quotient can now be easily calculated. We get that $\frac{\Lambda\left(V_{n}\right)}{(f)}(t)=\left\langle(1+t)^{n}\left(1-t^{2}\right)\right\rangle$, which is the same as the Hilbert series for the corresponding quotient in the square-free algebra.

Remark 5.1. It is not true that if $f_{e}=\sum_{1 \leqslant i<j \leqslant n} \alpha_{i j} x_{i} x_{j}$ is a non-generic quadratic form in $\bigwedge V_{n}$, and $f_{s}=\sum_{1 \leqslant i<j \leqslant n} \alpha_{i j} x_{i} x_{j}$ is the corresponding form in $\mathfrak{S}\left(V_{n}\right)$, then $\frac{\Lambda V_{n}}{\left(f_{e}\right)}$ and $\frac{\mathfrak{S}\left(V_{n}\right)}{\left(f_{s}\right)}$ have the same Hilbert series. For an example, consider the form $x_{1} x_{2}+x_{1} x_{3}+x_{1} x_{4}+x_{3} x_{4}$. The quotient of $\bigwedge V_{4}$ by this form has Hilbert series $5 t^{2}+4 t+1$, but the corresponding quotient of $\mathfrak{S}\left(V_{4}\right)$ has series $t^{3}+5 t^{2}+4 t+1$.

We next show that if the degree $d$ of $f$ is even, then the Hilbert series of the quotient $\frac{\wedge V_{n}}{(f)}$ is the same as for the square-free algebra. To this end, we need some combinatorial results, which we have collected in the appendix. With the aid of these, we can prove: 
Theorem 5.2. Let $f \in \wedge^{d} V$, with $d$ even, be a generic form. Then the linear transformation

$$
\wedge^{r} V \stackrel{f \cdot}{\longrightarrow} \wedge^{r+d} V
$$

is injective for $2 r+d \leqslant n$, and surjective for $2 r+d \geqslant n$.

Proof. We put $k=r+d$. Suppose that

$$
f=\sum_{K \in\left(\begin{array}{c}
{[n]} \\
d
\end{array}\right)} c_{K} x_{K}
$$

The matrix of the map (11) is an $\left(\begin{array}{c}n \\ r+d\end{array}\right) \times\left(\begin{array}{l}n \\ d\end{array}\right)$ matrix, $\widetilde{M_{r, r+d, n}}$, where the rows are indexed by $(r+d)$-subsets $K$, and the columns by $d$-subsets $T$. The entry at position $(K, T)$ is

$$
\begin{cases}0 & \text { if } T \nsubseteq K \\ \sigma(T, K) c_{T} & \text { if } T \subseteq K\end{cases}
$$

We must prove that this matrix has maximal rank. Clearly, the rank can not increase under specialisation, so if we prove that the matrix obtained by replacing each $c_{T}$ with 1 has maximal rank, then so does $\widetilde{M_{r, r+d, n}}$. However, the specialised matrix is nothing but the matrix $M_{r, r+d, n}$ of Theorem Appendix A.6, so it has full rank.

Theorem 5.3. Let $f \in \bigwedge V_{n}$ be a generic form of degree $d$, with $d$ even. Then

$$
\frac{\bigwedge V_{n}}{(f)}(t)=\left\langle(1+t)^{n}\left(1-t^{d}\right)\right\rangle=\delta_{n, d}(t)
$$

Proof. This follows from Theorem 5.2, together with (3).

\section{Principal ideals on generic forms of odd degree in the exterior algebra}

Let $d$ be an odd integer. Recall that we've conjectured that $a_{n, d}(t)-p_{n, d}(t) \rightarrow 0$ as $n \rightarrow \infty$, and that this conjecture leads to the conclusions that $p_{n, d}(t) \sim(1+$ $t)^{n}\left(1+t^{d}\right)^{-1}$. In this section, we shall try to guess the exact value of $q_{n, d}(t)$.

Since $a_{n, d}(t) \geqslant p_{n, d}(t), a_{n, d}(t) \geqslant \Delta_{n, d}(t)$, it follows that $a_{n, d}(t) \geqslant$ $\max \left(p_{n, d}(t), \Delta_{n, d}(t)\right)$. We tabulate the difference $a_{n, d}(t)-\max \left(p_{n, d}(t), \Delta_{n, d}(t)\right)$ in Table 2 and Table 3.

Using the data of Table 3, we make the following conjecture:

Conjecture 6.1. Let $d$ be an odd integer $>3$. Then, putting $\tau_{n, d}(t)=a_{n, d}(t)-$ $\max \left(p_{n, d}(t), \Delta_{n, d}(t)\right)$,

$$
\tau_{n, d}(t)= \begin{cases}t^{v(v-1) / 2} & \exists v, s \in \mathbb{N}: v>0, n-d=-1+\frac{5}{2} v+\frac{1}{2} v^{2}, d=5+2 v s \\ 0 & \text { otherwise }\end{cases}
$$


Table 2: Difference between true and predicted Hilbert series of the annihilator of a generic form of odd degree

\begin{tabular}{|c|l|c|c|c|c|c|c|c|c|}
\hline $\mathrm{n}$ & $\operatorname{deg}=3$ & 5 & 7 & 9 & 11 & 13 & 15 & 17 & 19 \\
\hline 3 & 0 & & & & & & & & \\
4 & 0 & & & & & & & & \\
5 & $\mathrm{t}$ & 0 & & & & & & & \\
6 & 0 & 0 & & & & & & & \\
7 & 0 & 0 & 0 & & & & & & \\
8 & 0 & 0 & $t$ & 0 & & & & & \\
9 & $3 t^{3}$ & 0 & 0 & 0 & & & & & \\
10 & $t^{4}$ & $t^{3}$ & 0 & $t$ & 0 & & & & \\
11 & $t^{5}$ & 0 & 0 & 0 & 0 & & & & \\
12 & $t^{6}+12 t^{5}$ & 0 & 0 & 0 & $t$ & 0 & & & \\
13 & $t^{7}+13 t^{6}+t^{5}$ & 0 & 0 & 0 & 0 & 0 & & & \\
14 & $t^{8}+14 t^{7}+91 t^{6}$ & 0 & 0 & $t^{3}$ & 0 & $\mathrm{t}$ & 0 & & \\
15 & $15 t^{8}+105 t^{7}$ & $t^{6}$ & 0 & 0 & 0 & 0 & 0 & & \\
16 & $16 t^{9}+120 t^{8}+559 t^{7}$ & & & 0 & 0 & $t$ & 0 & \\
17 & & & & 0 & 0 & 0 & 0 & 0 & \\
18 & & & & & & $t^{3}$ & 0 & $t$ & 0 \\
19 & & & & & & & 0 & 0 & 0 \\
20 & & & & & & & & 0 & $t$ \\
21 & & & & & & & & & \\
\hline
\end{tabular}

Table 3: Difference between true and predicted Hilbert series of the annihilator of a generic form of odd degree $>3$

\begin{tabular}{|c|c|c|c|c|c|c|c|c|}
\hline$n-d$ & $\operatorname{deg}=5$ & 7 & 9 & 11 & 13 & 15 & 17 & 19 \\
\hline 0 & 0 & 0 & 0 & 0 & 0 & 0 & 0 & 0 \\
\hline 1 & 0 & 0 & 0 & 0 & 0 & 0 & 0 & 0 \\
\hline 2 & $t$ & $t$ & $t$ & $t$ & $t$ & $t$ & $t$ & $t$ \\
\hline 3 & 0 & 0 & 0 & 0 & 0 & 0 & 0 & \\
\hline 4 & 0 & 0 & 0 & 0 & 0 & 0 & 0 & \\
\hline 5 & 0 & 0 & 0 & 0 & 0 & 0 & 0 & \\
\hline 6 & $t^{3}$ & 0 & $t^{3}$ & 0 & $t^{3}$ & & & \\
\hline 7 & 0 & 0 & 0 & 0 & & & & \\
\hline 8 & 0 & 0 & 0 & & & & & \\
\hline 9 & 0 & 0 & & & & & & \\
\hline 10 & 0 & & & & & & & \\
\hline 11 & $t^{6}$ & & & & & & & \\
\hline
\end{tabular}


This conjecture yields a formula for the Hilbert series, but since said formula is very complicated, we do not write it down; instead we show how to derive $q_{n, d}(t)$. From

$$
\begin{aligned}
& a_{n, d}(t)=\tau_{n, d}(t)+\max \left(p_{n, d}(t), \Delta_{n, d}(t)\right) \\
& q_{n, d}(t)=a_{n, d}(t) t^{d}+(1+t)^{n}\left(1-t^{d}\right) \\
& p_{n, d}(t)=(1+t)^{n}-q_{n, d}(t)
\end{aligned}
$$

we get

$$
\begin{aligned}
p_{n, d}(t) & =(1+t)^{n}-q_{n, d}(t) \\
& =(1+t)^{n}-a_{n, d}(t) t^{d}-(1+t)^{n}\left(1-t^{d}\right) \\
& =(1+t)^{n}-t^{d} \tau_{n, d}(t)-t^{d} \max \left(p_{n, d}(t), \Delta_{n, d}(t)\right)-(1+t)^{n}\left(1-t^{d}\right) \\
& =t^{d}\left((1+t)^{n}-\tau_{n, d}(t)-\max \left(p_{n, d}(t), \Delta_{n, d}(t)\right)\right)
\end{aligned}
$$

Hence, writing $p_{n, d}(t)=\sum_{i=0}^{n} a_{i} t^{i}$, with the $a_{i}$ 's as undetermined coefficients, and denoting the $t^{i}$-coefficient of $\tau_{n, d}(t)$ by $b_{i}$, we get the equation

$$
a_{\ell}=\left(\begin{array}{c}
n \\
\ell-d
\end{array}\right)-b_{i-\ell}-\max \left(a_{\ell-d},\left(\begin{array}{c}
n \\
\ell-d
\end{array}\right)-\left(\begin{array}{l}
n \\
\ell
\end{array}\right)\right)
$$

which we can solve recursively, using the initial values

$$
a_{0}=\cdots=a_{d-1}=0, \quad a_{d}=a_{n}=1 .
$$

For the case $d=3$, we proceed differently: we tabulate $q_{n, 3}(t)-w_{n, 3}(t)$ in Table 4 , and from that, make the following conjecture:

Conjecture 6.2. The Hilbert series of $\frac{\Lambda V_{n}}{(f)}$, where $f$ is a generic cubic form, is given by

$$
\begin{aligned}
p_{n, 3}(t) & =\frac{t^{d} L_{n}(t)+(1+t)^{n}}{1+t^{d}} \\
L_{n}(t) & = \begin{cases}(3 t)^{2 \ell-1}(1+t)^{2} & n=4 \ell \\
c_{1}(n) t^{2 \ell-1}(1+t)\left(1+\left(3^{c_{2}(n)}-1\right) t+t^{2}\right) & n=4 \ell+1 \\
(3 t)^{2 \ell}(1+t)^{2} & n=4 \ell+2 \\
(3 t)^{2 \ell+1}(1+t) & n=4 \ell+3\end{cases}
\end{aligned}
$$

where $c_{1}(n), c_{2}(n)$ are some positive integers.

\section{Hilbert series for generic non-principal ideals in the sym- metric and square-free algebra}

Let $I=\left(f_{1}, \ldots, f_{r}\right)$ be a generic ideals in $K\left[x_{1}, \ldots, x_{n}\right]$, generated by forms of degree $d_{1}, \ldots, d_{r}$. There is a famous conjecture $[\mathbf{1 7}, \mathbf{7}]$ for the Hilbert series of the quotient $\frac{K\left[x_{1}, \ldots, x_{n}\right]}{I_{n}}$. 


\begin{tabular}{|c|l|}
\hline $\mathrm{n}$ & $q_{n}(t)-w_{n}(t)$ \\
\hline 3 & $3 t(1+t)$ \\
4 & $3 t(1+t)^{2}$ \\
5 & $t(1+t)\left(t^{2}+8 t+1\right)$ \\
6 & $9 t^{2}(1+t)^{2}$ \\
7 & $27 t^{3}(1+t)$ \\
8 & $27 t^{3}(1+t)^{2}$ \\
9 & $3 t^{3}(1+t)\left(t^{2}+26 t+1\right)$ \\
10 & $81 t^{4}(1+t)^{2}$ \\
11 & $243 t^{5}(1+t)$ \\
12 & $243 t^{5}(1+t)^{2}$ \\
13 & $t^{5}(1+t)\left(t^{2}+728 t+1\right)$ \\
14 & $729 t^{6}(1+t)^{2}$ \\
15 & $2187 t^{7}(1+t)$ \\
16 & $2187 t^{7}(1+t)^{2}$ \\
\hline
\end{tabular}

Table 4: $a_{n, d}(t)-p_{n, d}(t)$ for a cubic generic form

Conjecture 7.1. Let $I=\left(f_{1}, \ldots, d_{r}\right) \subset K\left[x_{1}, \ldots, x_{n}\right]$ be a generic ideal, with $\left|f_{i}\right|=d_{1}$ for $1 \leqslant i \leqslant r$. Then the Hilbert series of the graded algebra $\frac{K\left[x_{1}, \ldots, x_{n}\right]}{I_{n}}$ is given by

$$
\left\langle(1-t)^{-n} \prod_{i=1}^{r}\left(1-t^{d_{i}}\right)\right\rangle
$$

It is easy to see that if $r \leqslant n$, the generators form a regular sequence, and hence that

$$
\frac{K\left[x_{1}, \ldots, x_{n}\right]}{I_{n}}(t)=(1-t)^{-n} \prod_{i=1}^{r}\left(1-t^{d_{i}}\right), \quad \text { for } n \geqslant r
$$

In particular, the conjecture holds for $r \leqslant n$. The conjecture is also know to be true for $r=n+1$.

We note that (21) implies that

$$
\lim _{n \rightarrow \infty} \frac{\frac{K\left[x_{1}, \ldots, x_{n}\right]}{I_{n}}(t)}{K\left[x_{1}, \ldots, x_{n}\right](t)}=\prod_{i=1}^{r}\left(1-t^{d_{i}}\right)
$$

Now suppose that $I=\left(f_{1}, \ldots, f_{r}\right)$ is a generic ideal in the square-free algebra, and that $f_{i}$ is a generic form of degree $d_{i}$. Then

$$
\frac{\mathfrak{S}\left(V_{n}\right)}{\left(f_{1}, \ldots, f_{r}\right)} \simeq \frac{K\left[x_{1}, \ldots, x_{n}\right]}{\left(f_{1}^{\prime}, \ldots, f_{r}^{\prime}, x_{1}^{2}, \ldots, x_{n}^{2}\right)}
$$

where $f_{i}^{\prime}$ can be taken to be a generic form in $K\left[x_{1}, \ldots, x_{n}\right]$ which maps to $f_{i}$ under the canonical epimorphism $K\left[x_{1}, \ldots, x_{n}\right] \rightarrow \mathfrak{S}\left(V_{n}\right)$. It seems reasonable to assume that the Hilbert series of the quotient will not change if we replace the squares of variables with generic quadratic forms. Conjecture 7.1 then leads to the following: 
Conjecture 7.2. Let $r, n, d_{1}, \ldots, d_{r}$, and let $I_{n}$ be a generic ideal $i \mathfrak{S}\left(V_{n}\right)$ with generators of degrees $d_{1}, \ldots, d_{r}$. Then

$$
\frac{\mathfrak{S}\left(V_{n}\right)}{I_{n}}(t)=\left\langle(1+t)^{n} \prod_{i=1}^{r}\left(1-t^{d_{i}}\right)\right\rangle
$$

If this conjecture holds (our computations support this), then it follows that

$$
\lim _{n \rightarrow \infty} \frac{\frac{\mathfrak{S}\left(V_{n}\right)}{I_{n}}(t)}{\mathfrak{S}\left(V_{n}\right)(t)}=\prod_{i=1}^{r}\left(1-t^{d_{i}}\right)
$$

This is analogous to (22).

\section{Hilbert series for generic non-principal ideals in the exte- rior algebra}

We now throw all caution to the wind to make some bold conjectures about the Hilbert series of non-principal generic ideals. Let $I_{n}=\left(f_{1}, \ldots, f_{r}\right)$ be a generic ideal in $\Lambda V_{n}$, with $\left|f_{i}\right|=d_{i}$, and consider the exact sequence

$$
0 \longrightarrow \operatorname{ann}\left(f_{r}\right)\left(-d_{r}\right) \longrightarrow \frac{\bigwedge V_{n}}{\left(f_{1}, \ldots, f_{r-1}\right)}\left(-d_{r}\right) \stackrel{\cdot f_{r}}{\longrightarrow} \frac{\bigwedge V_{n}}{\left(f_{1}, \ldots, f_{r-1}\right)} \longrightarrow \frac{\bigwedge V_{n}}{(I)} \longrightarrow 0
$$

We denote the Hilbert series of $\frac{\Lambda V_{n}}{(I)}$ by $q_{n}(t)$, that of $\frac{\Lambda V_{n}}{\left(f_{1}, \ldots, f_{r-1}\right)}$ by $u_{n}(t)$, and that of $\operatorname{ann}\left(f_{r}\right)$ by $a_{n}(t)$. Then

$$
q_{n}(t)=u_{n}(t)-t^{d_{r}} u_{n}(t)+t^{d} a_{n}(t) .
$$

If $d_{r}$ is even, we conjecture that $a_{n}(t) \sim 0$, hence

$$
q_{n}(t) \sim\left(1-t^{d_{r}}\right) u_{n}(t)
$$

If $d_{r}$ is odd, we conjecture that the annihilator of $f_{r}$ is "close" to the principal ideal on $f_{r}$, hence that $a_{n}(t) \sim\left(u_{n}(t)-q_{n}(t)\right)$, which yields

$$
q_{n}(t)\left(1+t^{d}\right) \sim u_{n}(t)
$$

By induction, we arrive at the following conjecture:

$$
\lim _{n \rightarrow \infty} \frac{q_{n}(t)}{(1+t)^{n}}=\prod_{i=1}^{r}\left(1-(-1)^{d_{i}} t^{d_{i}}\right)^{(-1)^{d_{i}}} \in \mathbb{Z}[[t]],
$$

with respect to the $(t)$-adic topology.

One would be tempted to guess that if all $d_{i}$ 's are even, the Hilbert series of $\frac{\wedge V_{n}}{\left(f_{1}, \ldots, f_{r}\right)}$ should be exactly

$$
(1+t)^{n} \prod_{i=1}^{r}\left(1-t^{d_{i}}\right)
$$

However, this is not true, even for the simplest case $r=2$ and $d_{1}=d_{2}=2$. In Table 5 we tabulate the difference between the true Hilbert series and (30). 


\begin{tabular}{|c|c|c|c|c|c|c|c|c|c|c|c|c|}
\hline $\mathrm{n}$ & 2 & 3 & 4 & 5 & 6 & 7 & 8 & 9 & 10 & 11 & 12 & 13 \\
\hline Diff & 0 & 0 & 0 & $t^{3}$ & 0 & $t^{4}$ & $t^{4}$ & $t^{5}$ & $10 t^{5}$ & $t^{6}+t^{5}$ & $64 t^{6}$ & $t^{7}+13 t^{6}$ \\
\hline
\end{tabular}

Table 5: Difference between the true Hilbert series and the "anticipated Hilbert series" for generic ideals generated by two quadratic forms

\section{Appendix A. The signed incidence matrix has full rank when the difference in cardinality is even}

We prove a "signed version" of the well-known theorem that the incidence matrix of $r$-subsets of $[n]=\{1, \ldots, n\}$ into $d+r$-subsets have full rank. Our proof is a modification of the one by Wilson $[\mathbf{1 8}]$.

To begin, let us define the "signs" involved.

Definition Appendix A.1. Let $[n]=\{1, \ldots, n\}$, and let $\mathcal{C}$ and $\mathcal{R}$ be two subsets of $[n]$, with

$$
\begin{aligned}
\mathcal{C} & =\left\{t_{1}, \ldots, t_{a}\right\}, \quad t_{1}<\cdots<t_{a} \\
\mathcal{R} & =\left\{k_{1}, \ldots, k_{b}\right\}, \quad k_{1}<\cdots<k_{b}
\end{aligned}
$$

Then define $\sigma(\mathcal{C}, \mathcal{R})$ to be zero if $\mathcal{C} \nsubseteq \mathcal{R}$, and otherwise the sign of the permutation which sorts $[\mathcal{C}, \mathcal{R} \backslash \mathcal{C}]$ in ascending order. In other words, if $\mathcal{C} \subseteq \mathcal{R}$ then $\sigma(\mathcal{C}, \mathcal{R})$ is the sign of the uniquely determined permutation $\gamma$ such that

$$
\begin{aligned}
t_{\gamma(i)} & =k_{i}, \quad 1 \leqslant i \leqslant a \\
k_{\gamma(j)} & =k_{a+j}, \quad 1 \leqslant j \leqslant b
\end{aligned}
$$

Definition Appendix A.2. Let $[n]=\{1, \ldots, n\}$, and let $A, B$ be two subsets of $[n]$, of cardinality $a$ and $b$, with $0 \leqslant a<b$. For $a \leqslant r<b$, we define

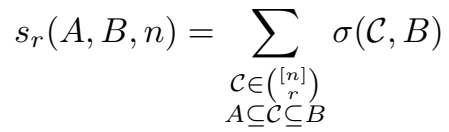

For $0 \leqslant d \leqslant n$, we define

$$
s_{d, n}=\sum_{R \in\left(\begin{array}{c}
{[n]} \\
d
\end{array}\right)} \sigma(R,[n])=s_{d}(\emptyset,[n], n)
$$

Lemma Appendix A.3. With the notations of Definition Appendix A.2, put $d=$ $b-r$. We have that

$$
s_{r}(A, B, n)= \begin{cases}0 & A \nsubseteq \subseteq B \\ (-1)^{d} s_{d, b-a} & A \subseteq B\end{cases}
$$

Proof. Put $d=b-r$. If $A \nsubseteq B$ then clearly $s_{r}(A, B, n)=0$. Suppose that $A \subseteq B$. Then

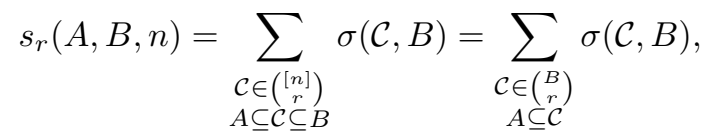


so the sum is independent of $n$. Furthermore, we can write $A \subseteq \mathcal{C} \in\left(\begin{array}{l}B \\ r\end{array}\right)$ as a disjoint union $\mathcal{C}=A \cup(\mathcal{C} \backslash A)$, hence the sum can be written

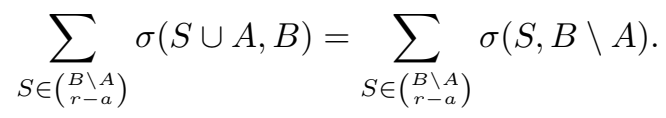

Now, since $S$ has cardinality $r-a$, the set $(B \backslash A) \backslash S$ has cardinality $b-a-(r-a)=$ $b-r=d$, so the permutation which transforms $[S, B \backslash A]$ to $[B \backslash A, S]$ has cardinality $(-1)^{d}$. Hence, by substituting $R=(B \backslash A) \backslash S$, we get that the sum is equal to

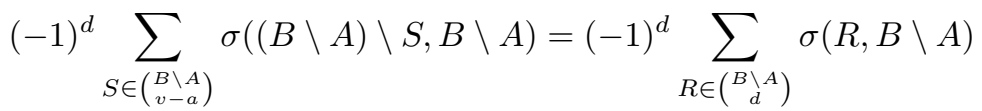

$$
\begin{aligned}
& =(-1)^{d} \sum_{R \in\left(\begin{array}{c}
{[b-a]} \\
d
\end{array}\right)} \sigma(R,[b-a]),
\end{aligned}
$$

which is the desired result.

Lemma Appendix A.4. Suppose that $0<d \leqslant n$, and that $d$ is even. Then $s_{d, n}>0$.

Proof. The lemma is trivially true for $d=n$. If $d=2$, we note that $\sigma(\{v, v+1\},[n])=$ 1 for $1 \leqslant v<n$, since the permutation transforming $[v, v+1,1,2, \ldots, v-1, v+2, v+$ $3, \ldots, n]$ to $[1,2, \ldots, n]$ is even. Furthermore, the signs of $\sigma(\{v, v+\ell\},[n])$ alternate in sign as $\ell$ goes from 1 to $n-v$. Thus, for a fixed $v$, there are either as many positive as negative $\sigma(\{v, v+\ell\},[n])$, or 1 more positive than negative, depending on the parity of $n-v$. By summing over all $v$, we conclude that there are always strictly more positive than negative signs.

Now suppose that we have shown that $s_{2 k^{\prime}, n^{\prime}}>0$ for all $k^{\prime}, n^{\prime}$ such that $k^{\prime}<k$. We want to show that that $s_{2 k, n}>0$. We have that

$$
s_{2 k, n}=\sum_{R \in\left(\begin{array}{c}
{[n]} \\
2 k
\end{array}\right)} \sigma(R,[n]),
$$

and writing $R$ as a disjoint union of its first two element, and the remaining elements, this becomes

$$
\begin{aligned}
& \sum_{1 \leqslant k<\ell \leqslant n-2} \sum_{R_{2} \in\left(\begin{array}{c}
\{\ell+1, \ell+2, \ldots, n\} \\
2 k-2
\end{array}\right)} \sigma\left(\{k, \ell\} \cup R_{2},[n]\right) \\
& =\sum_{1 \leqslant k<\ell \leqslant n-2} \sum_{R_{2} \in\left(\begin{array}{c}
\{\ell+1, \ell+2, \ldots, n\} \\
2 k-2
\end{array}\right)} \sigma\left(R_{2},\{\ell+1, \ell+2, \ldots, n\}\right) \\
& =\sum_{1 \leqslant k<\ell \leqslant n-2} s_{2 k-2, n-\ell}>0 .
\end{aligned}
$$

Next, we define the signed incidence matrix. 
Definition Appendix A.5. Let $0<a<b \leqslant n$ be integers. Then $M_{a, b, n}$ is the $\left(\begin{array}{l}n \\ b\end{array}\right) \times\left(\begin{array}{l}n \\ a\end{array}\right)$ matrix where the rows are indexed by $b$-subsets of $[n]$, the columns by $a$-subsets of $[n]$, and where the entry in row $B$, column $A$ is $\sigma(A, B)$.

Theorem Appendix A.6. Let $0<a<b \leqslant n$ be integers. If $d=b-a$ is even, then $M_{a, b, n}$ has full rank.

Proof. Denote the row indexed by $\mathcal{R} \in\left(\begin{array}{c}{[n]} \\ b\end{array}\right)$ by $\tau_{\mathcal{R}}$, then $\tau_{\mathcal{R}}$ can be regarded as an element in $V_{a}([n])$, the free $\mathbb{Q}$-vector space on the $a$-subsets of $[n]$. If we denote the basis element corresponding to a $a$-subset $\mathcal{C}$ by $\epsilon_{\mathcal{C}}$, then

$$
\tau_{\mathcal{R}}=\sum_{\mathcal{C} \in\left(\begin{array}{c}
{[n]} \\
a
\end{array}\right)} \sigma(\mathcal{C}, \mathcal{R}) \epsilon_{\mathcal{C}}
$$

The number of rows in $M_{a, b, n}$ is $\left(\begin{array}{l}n \\ b\end{array}\right)$, and the number of columns is $\left(\begin{array}{l}n \\ a\end{array}\right)$. There are less rows than columns if $a+b>n$, as many rows as columns if $a+b=n$, and more rows than columns if $a+b<n$.

1. If $a+b \geqslant n$, we must prove that the rows are linearly independent. Suppose that there is a linear relation among the $\tau_{\mathcal{R}}$ 's, so that

$$
\sum_{\mathcal{R} \in\left(\begin{array}{c}
{[n]} \\
b
\end{array}\right)} a_{\mathcal{R}} \tau_{\mathcal{R}}=0
$$

for some numbers $a_{\mathcal{R}}$. We shall prove that all $a_{\mathcal{R}}=0$.

Choose an $I \subset\left(\begin{array}{c}{[n]} \\ i\end{array}\right), 0 \leqslant i \leqslant a$, and define a linear functional $H_{I}: V_{a}([n]) \rightarrow \mathbb{Q}$ by

$$
f_{I}\left(\epsilon_{\mathcal{C}}\right)= \begin{cases}1 & I \subseteq \mathcal{C} \\ 0 & I \nsubseteq \mathcal{C}\end{cases}
$$

Then if $\mathcal{R} \in\left(\begin{array}{c}{[n]} \\ b\end{array}\right)$ we have that

$$
\begin{aligned}
f_{I}\left(\tau_{\mathcal{R}}\right)=f_{I}\left(\sum_{\mathcal{C} \in\left(\begin{array}{c}
{[n]} \\
a
\end{array}\right)} \sigma(\mathcal{C}, \mathcal{R}) \epsilon_{\mathcal{C}}\right)=\sum_{\mathcal{C} \in\left(\begin{array}{c}
{[n]} \\
a
\end{array}\right)} \sigma(\mathcal{C}, \mathcal{R}) f_{I}\left(\epsilon_{\mathcal{C}}\right) \\
\quad=\sum_{I \subseteq \mathcal{C} \subseteq \mathcal{R}} \sigma(\mathcal{C}, \mathcal{R})=s_{a}(I, \mathcal{R}, n)= \begin{cases}s_{d, b-i} & I \subseteq \mathcal{R} \\
0 & I \nsubseteq \mathcal{R}\end{cases}
\end{aligned}
$$

The last step follows from Lemma Appendix A.3. Applying $f_{I}$ to (34) we get that

$$
\begin{aligned}
0=f_{I}\left(\sum_{\mathcal{R} \in\left(\begin{array}{c}
{[n]} \\
b
\end{array}\right)} a_{\mathcal{R}} \tau_{\mathcal{R}}\right) & =\sum_{\mathcal{R} \in\left(\begin{array}{c}
{[n]} \\
b
\end{array}\right)} a_{\mathcal{R}} f_{I}\left(\tau_{\mathcal{R}}\right) \\
& =\sum_{\mathcal{R} \in\left(\begin{array}{c}
n n] \\
b
\end{array}\right)} a_{\mathcal{R}} s_{a}(I, \mathcal{R})=s_{d, b-i} \sum_{\mathcal{R} \in\left(\begin{array}{c}
{[n]} \\
b
\end{array}\right) \mathcal{R} \supseteq I} a_{\mathcal{R}}
\end{aligned}
$$


Since Lemma Appendix A.4 tells us that $s_{d, b-i} \neq 0$, we conclude that

$$
\sum_{\mathcal{R} \supseteq I} a_{\mathcal{R}}=0
$$

Now, for any $J \subset[n]$ we have, by exclusion-inclusion, that

$$
\sum_{\mathcal{R} \cap J=\emptyset} a_{\mathcal{R}}=\sum_{I \subset J}(-1)^{|I|} \sum_{\mathcal{R} \supseteq I} a_{\mathcal{R}}
$$

Fix $\mathcal{R}_{0} \in\left(\begin{array}{c}{[n]} \\ b\end{array}\right)$ and put $J_{0}=[n] \backslash \mathcal{R}_{0}$. Since $\left|J_{0}\right|=n-b \leqslant a$ we have, using (38) that

$$
a_{\mathcal{R}_{0}}=\sum_{\mathcal{R} \cap J_{0}=\emptyset} a_{\mathcal{R}}=\sum_{I \subseteq J_{0}}(-1)^{|I|} \sum_{\mathcal{R} \supseteq I} a_{\mathcal{R}}=0
$$

Since $a_{\mathcal{R}_{0}}$ was arbitrary, all $a_{\mathcal{R}}$ are zero. This shows that the $\tau_{\mathcal{R}}$ are linearly independent.

2. If $\mathrm{n}=\mathrm{a}+\mathrm{b}$, then $M$ is a square matrix. By the previous case, the vectors $\tau_{\mathcal{R}}$ are linearly independent, but since there are $\left(\begin{array}{l}n \\ a\end{array}\right)=\left(\begin{array}{l}n \\ b\end{array}\right)$ such vectors, they form a basis of $V_{a}([n])$; in particular, they span this vector space.

3. Finally, let us consider the remaining case $n>a+b$, so that there are more rows than columns. We must prove that the rows span $V_{a}([n])$. We prove this by induction over $n-a-b$. The case $n-a-b=0$ is already proved, and forms the basis of the induction. We assume $a, b$ fixed, and that the assertion has been proved for all $a+b \leqslant n^{\prime}<n$.

Let $\Gamma \in\left(\begin{array}{c}{[n]} \\ a\end{array}\right)$ be arbitrary. If we can express $\alpha=\epsilon_{\Gamma}$ as a linear combination of the $\tau_{\mathcal{R}}$ 's, we are done. To this end, put

$$
\alpha^{\prime}=\sum_{\substack{S \in([n-1] \\ a-1 \\ S \cup\{n\}=\Gamma}} \epsilon_{S} \in V_{a-1}([n-1])
$$

Since $a-1+b<n-1$, it follows by induction that there are scalars $\left\{d_{J} \mid J \in\left(\begin{array}{c}{[n-1]} \\ a-1\end{array}\right)\right\}$ such that

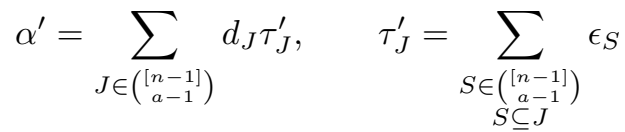

For $\mathcal{R} \in\left(\begin{array}{c}{[n]} \\ a\end{array}\right), n \in \mathcal{R}$, put $c_{\mathcal{R}}^{\prime}=d_{\mathcal{R}} \backslash\{n\}$. Define

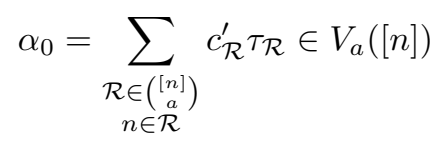

If we write

$$
\alpha_{0}=\sum_{\mathcal{C} \in\left(\begin{array}{c}
{[n]} \\
a
\end{array}\right)} a_{\mathcal{C}}^{\prime} \epsilon_{\mathcal{C}}
$$


we have that for $\mathcal{C} \in\left(\begin{array}{c}{[n]} \\ a\end{array}\right), n \in \mathcal{C}$, that

$$
a_{\mathcal{C}}^{\prime}= \begin{cases}1 & \mathcal{C}=\Gamma \\ 0 & \mathcal{C} \neq \Gamma\end{cases}
$$

which implies that

$$
\alpha_{0}= \begin{cases}\alpha & n \in \Gamma \\ 0 & n \notin \Gamma\end{cases}
$$

In either case, $\alpha-\alpha_{0}$ has coordinate 0 in component $\mathcal{R} \in\left(\begin{array}{c}{[n]} \\ a\end{array}\right)$, unless $n \in \mathcal{R}$. Hence, $\alpha-\alpha_{0}$ may be regarded as a vector in $V_{a}([n-1])$. By the induction hypothesis, there exist $c_{\mathcal{R}}^{\prime \prime}$ such that

$$
\alpha-\alpha_{0}=\sum_{\mathcal{R} \in\left(\begin{array}{c}
{[n-1]} \\
a
\end{array}\right)} c_{\mathcal{R}}^{\prime \prime} \tau_{\mathcal{R}}
$$

Defining

$$
c_{\mathcal{R}}= \begin{cases}c_{\mathcal{R}}^{\prime} & n \in \mathcal{R} \\ c_{\mathcal{R}}^{\prime \prime} & n \notin \mathcal{R}\end{cases}
$$

we get that

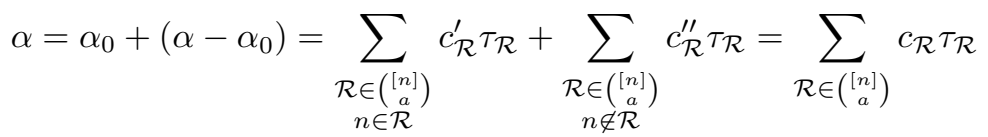

\section{Appendix B. Calculations}

The computer calculations were done on the computers of the UMS Medicis, cole Polytechnique, and on the computers at the Department of Mathematics, Stockholm University. We have used the programme Macaulay $2[\mathbf{1 2}]$ to calculate Hilbert series and minimal free resolutions. To save time and memory, the calculations were performed in characteristic 31991. The holes in the tables show that there are limits to what we could calculate, even on a machine with 2 GB of memory. 


\section{References}

[1] Anetta Aramova, Luchezar L. Avramov, and Jürgen Herzog. Resolutions of monomial ideals and cohomology over exterior algebras. Transactions of the American Mathematical Society, 352(2):579-594, 1999.

[2] Annetta Aramova, Jürgen Herzog, and Takayuki Hibi. Gotzman theorems for exterior algebras and combinatorics. Journal of Algebra, 191:174-211, 1997.

[3] A. M. Bigatti. Aspetti Combinatorici e Computazionali dell'Algebra Commutativa. PhD thesis, Università di Torino, 1995.

[4] N. Bourbaki. Éléments de mathématique. Première partie: Les structures fondamentales de l'analyse. Livre II: Algèbre. Chapitre 9: Formes sesquilinéaires et formes quadratiques. Hermann, Paris, 1959. Actualités Sci. Ind. no. 1272.

[5] G. F. Clements and B. Lindström. A generalization of a combinatorial theorem of Macaulay. J. Combinatorial Theory, 7:230-238, 1969.

[6] David Eisenbud. Commutative Algebra with a View Toward Algebraic Geometry, volume 150 of Graduate Texts in Mathematics. Springer Verlag, 1995.

[7] Ralf Fröberg. An inequality for Hilbert series of graded algebras. Mathematica Scandinavica, 56:117-144, 1985.

[8] Ralf Fröberg and Joachim Hollman. Hilbert series for ideals generated by generic forms. Journal of Symbolic Computation, 17:149-157, 1994.

[9] Ralf Fröberg and Clas Löfwall. On Hilbert series for commutative and noncommutative graded algebras. Journal of Pure and Applied Algebra, 76:33-38, 1991. North Holland.

[10] Ralf Fröberg and Clas Löfwall. Koszul homology and lie algebras with application to generic forms and points. Technical Report 8, Department of Mathematics, Stockholm University, 2000.

[11] J. E. Graver and W. B. Jurkat. The module structure of integral designs. Journal of Combinatorial Theory. Series A, 15:75-90, 1973.

[12] Daniel R. Grayson and Michael E. Stillman. Macaulay 2. Computer algebra program, available at http://www .math.uiuc.edu/Macaulay2/.

[13] William M. Kantor. On incidence matrices of finite projective and affine spaces. Mathematische Zeitschrift, 124:315-318, 1972.

[14] G. Katona. A theorem of finite sets. In Theory of graphs (Proc. Colloq., Tihany, 1966), pages 187-207. Academic Press, New York, 1968.

[15] Joseph B. Kruskal. The number of simplices in a complex. In Mathematical optimization techniques, pages 251-278. Univ. of California Press, Berkeley, Calif., 1963.

[16] F. S. Macaulay. Some properties of enumeration in the theory of modular systems. Proceedings of the London Mathematical Society, 26:531-555, 1927. 
[17] Guillermo Moreno-Socías. Autour de la fonction de Hilbert-Samuel (escaliers d'idéaux polynomiaux). PhD thesis, École Polytechnique, 1991.

[18] Richard M. Wilson. The necessary conditions for $t$-designs are sufficient for something. Utilitas Mathematica, 4:207-215, 1973.

This article may be accessed via WWW at http://www.rmi.acnet.ge/hha/ or by anonymous ftp at

ftp://ftp.rmi.acnet.ge/pub/hha/volumes/2002/n2a18/v4n2a18.(dvi,ps,pdf)

Guillermo Moreno-Socías moreno@gage.polytechnique.fr

Laboratoire GAGE,

cole Polytechnique

91128 Palaiseau Cedex

France

Jan Snellman jasne@mai.liu.se

Department of Mathematics

Linköping University

SE-58183 Linköping

SWEDEN 